\title{
Updating velocity models for complex pre-salt targets in Santos basin
}

\author{
Sergio Barragan, Daniela Donno, Florian Jouno, Adriano Martinez and Adel Khalil, CGG
}

Copyright 2019, SBGf - Sociedade Brasileira de Geofísica

This paper was prepared for presentation during the $16^{\text {th }}$ International Congress of the Brazilian Geophysical Society held in Rio de Janeiro, Brazil, 19-22 August 2019.

Contents of this paper were reviewed by the Technical Committee of the $16^{\text {th }}$ International Congress of the Brazilian Geophysical Society and do not necessarily represent any position of the SBGf, its officers or members. Electronic reproduction or storage of any part of this paper for commercial purposes without the written consent of the Brazilian Geophysical Society is prohibited.

\begin{abstract}
Pre-salt oil discoveries in the Santos basin have made it one of the most prospective petroleum provinces in the world. The prospects that these plays provide are not without challenge. Complex geological features and deep targets complicate the exploration and evaluation process. Limited illumination of available narrow-azimuth (NAZ) seismic data further add to the challenge. Full waveform inversion (FWI) is a natural choice to update complex overburdens. The recently proposed formulation by Zhang et al. (2018) provides a reliable approach for the complex geologies of the Santos basin. For deeper updates, waveequation migration velocity analysis (WEMVA) relies on reflections through the wave-equation, making it a potential candidate for salt and pre-salt model updates. While still susceptible to the limitations imposed by NAZ data, Donno et al. (2019) introduced a regularization to reduce some of these adverse effects. Iterating these two flavors of waveequation based inversions, we demonstrate that reasonable velocity updates can be achieved in some of the complex areas of the Santos basin using NAZ data. Salt delineation and scenario testing are still required in some places to improve unresolved areas. Results are promising with a significant uplift in the pre-salt layer.
\end{abstract}

\section{Introduction}

Pre-salt oil discoveries in the Santos basin have made it one of the most prospective petroleum provinces in the world. Since the first discovery by Petrobras in 2006, presalt contributes around $48 \%$ of Brazil's oil national production. The prospects that these plays provide are not without challenge. Complex geological features and deep targets complicate the exploration and evaluation process.

Limited illumination of available narrow-azimuth (NAZ) seismic data over Santos basin further add to the challenge. Overhangs, the base of salt and the pre-salt layer are often poorly imaged, rendering interpretation difficult. Diving wave penetration is limited by the relatively short offsets. The narrow illumination range and lack of low frequencies restrict the reliability of velocity updates.
Full waveform inversion (FWI) is a natural choice to update complex overburdens, however, classical formulations may fail in challenging environments as in the Santos basin due to the presence of salt, carbonates, volcanic intrusions and abundance of overhangs. The time-lag FWI (TLFWI) formulation recently proposed by Zhang et al. (2018) provides a more reliable alternative, as it relies on a traveltime misfit function which is less affected by the approximation made to the modeling engine.

For deeper updates, ray-based reflection tomography has been the industry standard, however, the high-frequency assumption often breaks under this complex geological context. Alternatively, reflection full waveform inversion (RFWI) relies directly on the wave-equation, making it more suitable for complex geologies; however, it suffers from amplitude mismatch between real data and modeled synthetic data. Image-based methods such as waveequation migration velocity analysis (WEMVA) depend more on the kinematic part of the propagated wavefields. While still susceptible to the restrictions imposed by NAZ data, Donno et al. (2019) proposed a regularized stackpower WEMVA formulation, which reduces the footprint of NAZ acquisition geometry upon the velocity updates..

Iterating the two flavors of wave-equation based inversion methods, i.e. TLFWI and stack-power WEMVA, we demonstrate that reasonable velocity updates can be achieved in some of the complex areas of the deep-water Santos basin, where volcanic intrusions, carbonates and diverse salt shapes are present. We apply the proposed workflow on a field NAZ dataset. Salt delineation and scenario testing are still required in some places to improve unresolved areas. Results are promising with a significant uplift in the base-of-salt and pre-salt layer.

\section{Time-lag FWI}

In the Santos basin, carbonates, volcanic intrusions and salt bodies, which often feature in complex geometries, are particularly troublesome for FWI. In the presence of sharp contrasts, such as a sediment-salt interface, small salt mispositionings create large traveltime and amplitude differences between modeled and observed data. As a consequence, extensive testing is required to establish the correct salt geometry of the initial velocity model, to mitigate the risk of FWI converging into a local minimum.

Several methods have been proposed to deal with cycle skipping and amplitude discrepancy between modeled and observed data (e.g., Ma \& Hale, 2013; Warner \& Guasch, 2016). Following the approach proposed by Luo \& Schuster (1991), TLFWI attempts to minimize the traveltime difference between observed and modeled data, and uses a cross-correlation coefficient weight to promote 

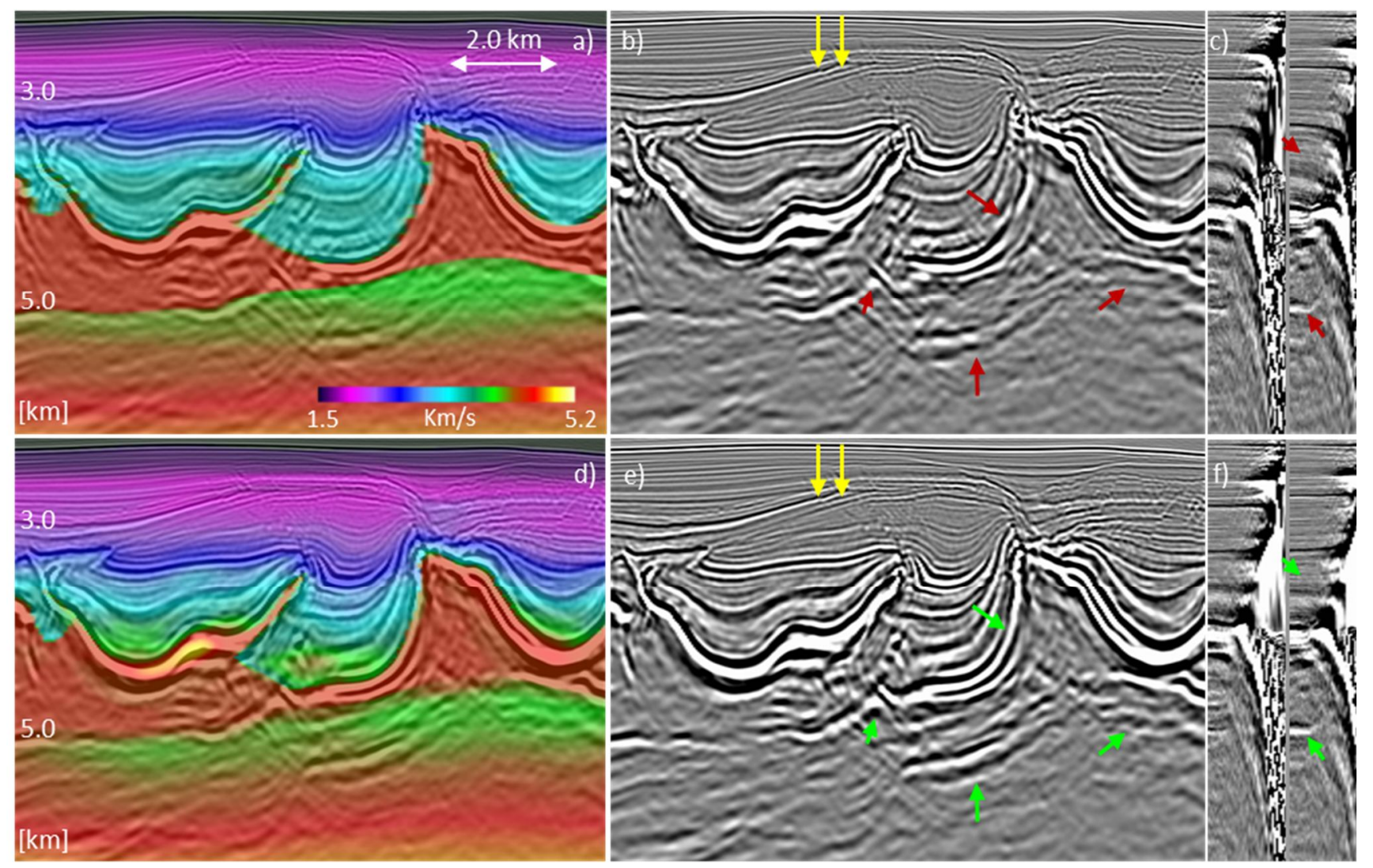

Figure 1 - Section showing the application of TLFWI. (a) Legacy model obtained with conventional flow, and (b) corresponding RTM stack image. (d) Updated velocity model after TLFWI using a maximum frequency of $10 \mathrm{~Hz}$, and (e) corresponding RTM stack image. Yellow arrows indicate the location of Kirchhoff CIGs for (c) legacy model and (f) TLFWI updated model. Complex top-of-salt thrust finger and mini-basins are well resolved despite the use of NAZ data.

the more reliable traveltime measurements during the inversion (Zhang et al., 2018). The traveltime difference is computed by maximizing the cross-correlation between observed and modeled data in frequency-dependent time windows. As a result, this formulation is largely driven by the wavefield kinematics. Amplitude differences due to sharp velocity contrasts or missing physics in the modeling engine have less impact on the stability of TLFWI formulation, compared to conventional FWI.

An example of the application of TLFWI in the presence of complex salt bodies is shown in Figure 1. The input data were acquired using a NAZ acquisition with variable-depth streamers, having a maximum offset of $8.2 \mathrm{~km}$ along the cables and $1.1 \mathrm{~km}$ across the cables. Data preparation for TLFWI includes mild noise attenuation, anti-alias filtering and resampling. The source wavelet is estimated from the direct arrival by solving a linear inverse problem (Pratt, 1999).

The starting velocity model is a smoothed version of the legacy model (Figure 1a). The legacy model was obtained through several iterations of ray-based reflection tomography and conventional FWI for the shallow sediments, top-down salt interpretation, ray-based reflection tomography at overhang levels and ray-based tomography for the pre-salt. The conventional model building sequence fails at capturing the complexity of the geology with the limitations given by the NAZ acquisition. Referring to the RTM stack image and Kirchhoff gathers obtained with the legacy model (Figures $1 \mathrm{~b}$ and 1c), we can observe that the complexities of the overburden are not fully resolved, and this has a direct impact on the poor imaging of the deeper layers.

We updated the velocity model using TLFWI starting from $3.5 \mathrm{~Hz}$ and reaching a maximum frequency of $10 \mathrm{~Hz}$. The updated velocity model is shown in Figure $1 \mathrm{~d}$. We can observe that the overburden sediments appear more focused in the RTM stack image (Figure 1e), mostly around the salt thrust finger and the mini-basin flanks. Moreover, the updated sediment velocity at overhangs and minibasins is geologically consistent, producing flatter gathers (Figure 1f). The improvements in the overburden also brought significant image uplift in the pre-salt layer.

The velocity update of TLFWI is mostly driven by diving waves. With a maximum offset of $8.2 \mathrm{~km}$, the penetration depth of diving waves is limited, hence, the maximum effectiveness of the algorithm is focused mostly on postsalt and intra-salt updates. For the pre-salt, velocity updates have to rely on reflection data. 

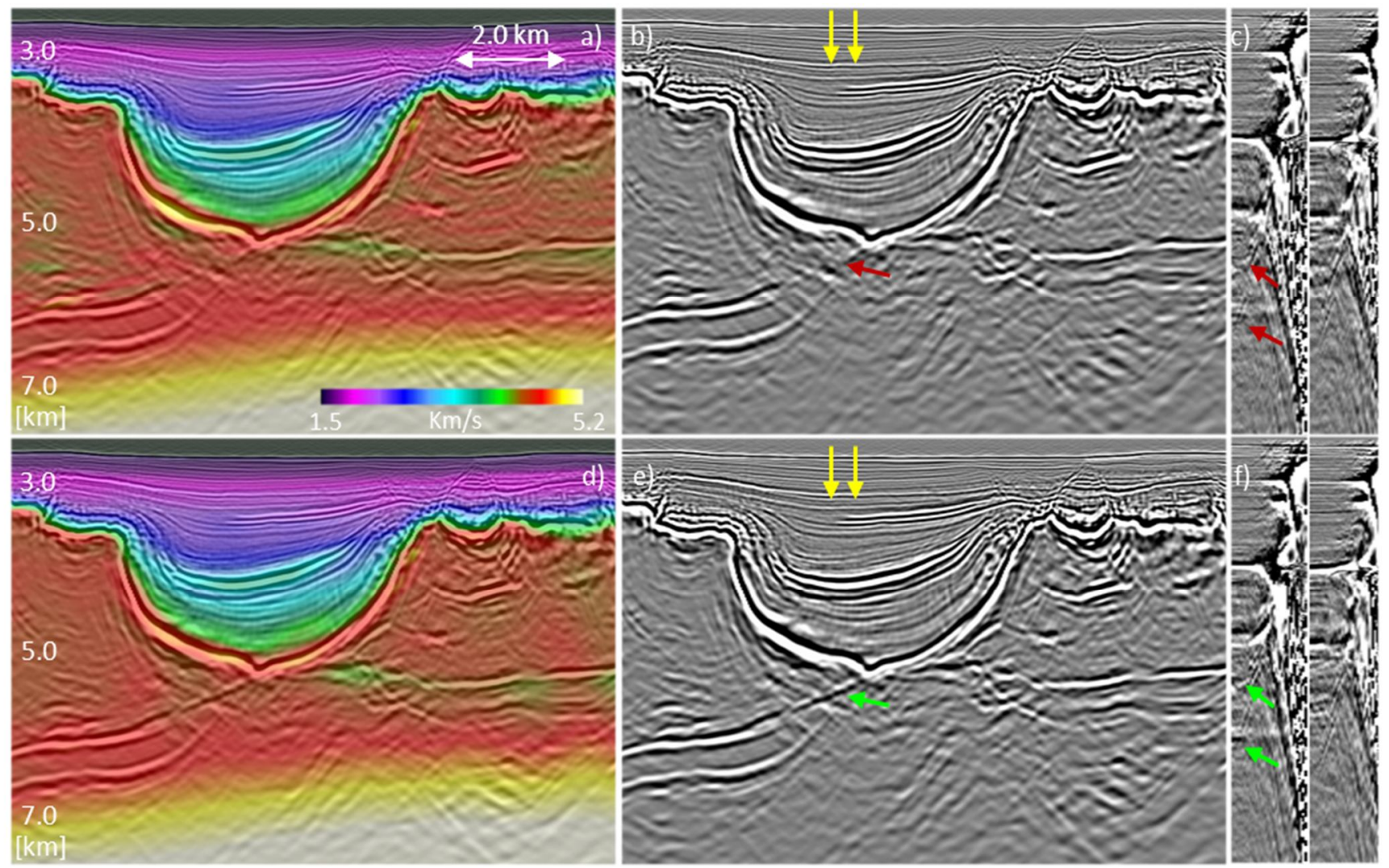

Figure 2 - Section showing the application of IRFWI. (a) Velocity model after TLFWI for post-salt sediments and shallow parts of salt, and (b) corresponding RTM stack image. (d) Updated velocity model using stack-power WEMVA and (e) corresponding RTM stack image. Yellow arrows indicate CIG positions for (c) TLFWI update and (f) TLFWI+WEMVA updated model. Base of salt appears more focused.

\section{Stack-power WEMVA}

RFWI (Xu et al., 2012) is effective at providing velocity updates beyond the penetration depth of diving waves. Recent applications of velocity inversion methods driven by RFWI have been implemented by Irabor \& Warner (2016), Sun et al. (2016), Gomes \& Chazalnoel (2017), Gomes \& Yang (2018) with promising results. The essence of this method lies in iterating between a migration phase and a tomographic phase, where the migration phase is used to model the synthetic reflected data, and the tomographic phase allows to estimate low-wavenumber updates of the velocity models. Nonetheless, this methodology often faces the difficulty of amplitude mismatch between the observed and modeled synthetic reflection data.

To mitigate the adverse effects of amplitude variations, Donno et al. (2019) presented the regularized stack-power WEMVA. This method uses an objective function defined in the image domain, as proposed by Soubaras \& Gratacos (2007), which is less sensitive to amplitude discrepancy than data-based methods. Stack-power WEMVA has a tomographic kernel similar to that of RFWI, which guarantees deeper penetration of the velocity updates when compared to refracted or diving waves. Moreover, the use of a regularization term allows mitigation of footprints created in the velocity updates when using NAZ data.

Stack-power WEMVA was applied in our workflow starting from $5 \mathrm{~Hz}$ and reaching a maximum frequency of $7 \mathrm{~Hz}$. Compared to TLFWI, a lower frequency bandwidth was chosen for the inversion because the objective was to update the deepest sections of salt and pre-salt layers. The pre-processing of the input seismic data included denoise, deghost, surface-related multiple elimination and shot regularization. Moreover, diving waves were muted so as to consider only reflections.

Reasonable results were obtained after several iterations of stack-power WEMVA velocity update using the previous overburden TLFWI update as input velocity model (Figure $2 a)$. Figures $2 b$ and $2 c$ show the corresponding RTM stack image and CIG gathers. As shown in Figure 2d, the velocity change in the updated model is not very large, however, the impact on the image is considerable. We can observe that, after the WEMVA update, the base of salt is more focused and more continuous (Figure $2 \mathrm{e}$ and 2f), therefore, it can be interpreted with greater certainty. 

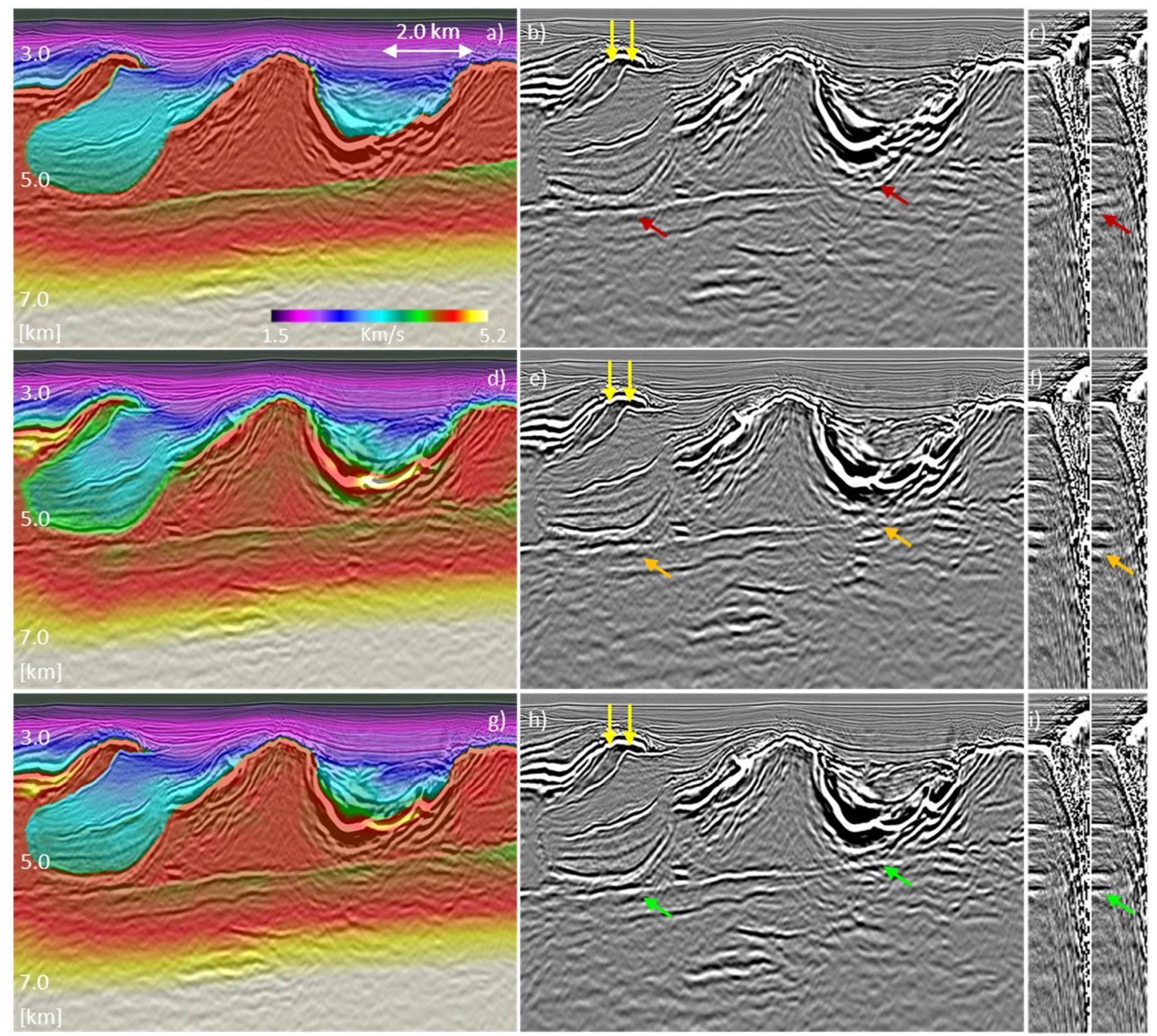

Figure 3 - Section showing the results of the iterative approach. $(a, b, c)$ Legacy model, RTM stack image and CIGs; $(d, e, f)$ updated velocity model, RTM stack image and CIGs after first iteration of TLFWI and stack-power WEMVA; $(g, h$, i) updated velocity model, RTM stack image and CIGs after second iteration of TLFWI and stack-power WEMVA. Yellow arrows indicate CIG position.

\section{Iterating TLFWI and stack-power WEMVA}

Using the two flavours of wave-equation based methods presented in the previous sections, we propose an iterative approach that alternates the application of TLFWI for updating the post-salt and the intra-salt and the application of stack-power WEMVA for those deep layers sampled only by reflections. The advantage of an iterative approach is that it allows mitigating some of the non-linearities of the model building process, related for example with the use of different types of waves (diving and reflection) in the two used inversion methods. This leads to a better inversion than what would be achieved by simple sequential application of TLFWI and stack-power WEMVA. We believe this is particularly true when dealing with NAZ data with $8 \mathrm{~km}$ of maximum offset, with a higher risk of poorly constrained model inversion.

Figure 3 shows an example of the improvement brought by the iterative approach. The legacy velocity model is shown in Figure 3a, with the corresponding RTM stack image and gathers in Figures $3 b$ and $3 c$, respectively. After the first pass of TLFWI and stack-power WEMVA, we can note several velocity updates (Figure $3 d$ ) from the post-salt down to the pre-salt level. The resulting stack image and gathers (Figure $3 e$ and $3 \mathrm{f}$ ) are improved in terms of better focusing and continuity of the events at the base-of-salt level and in the pre-salt layer. After the second iteration of 

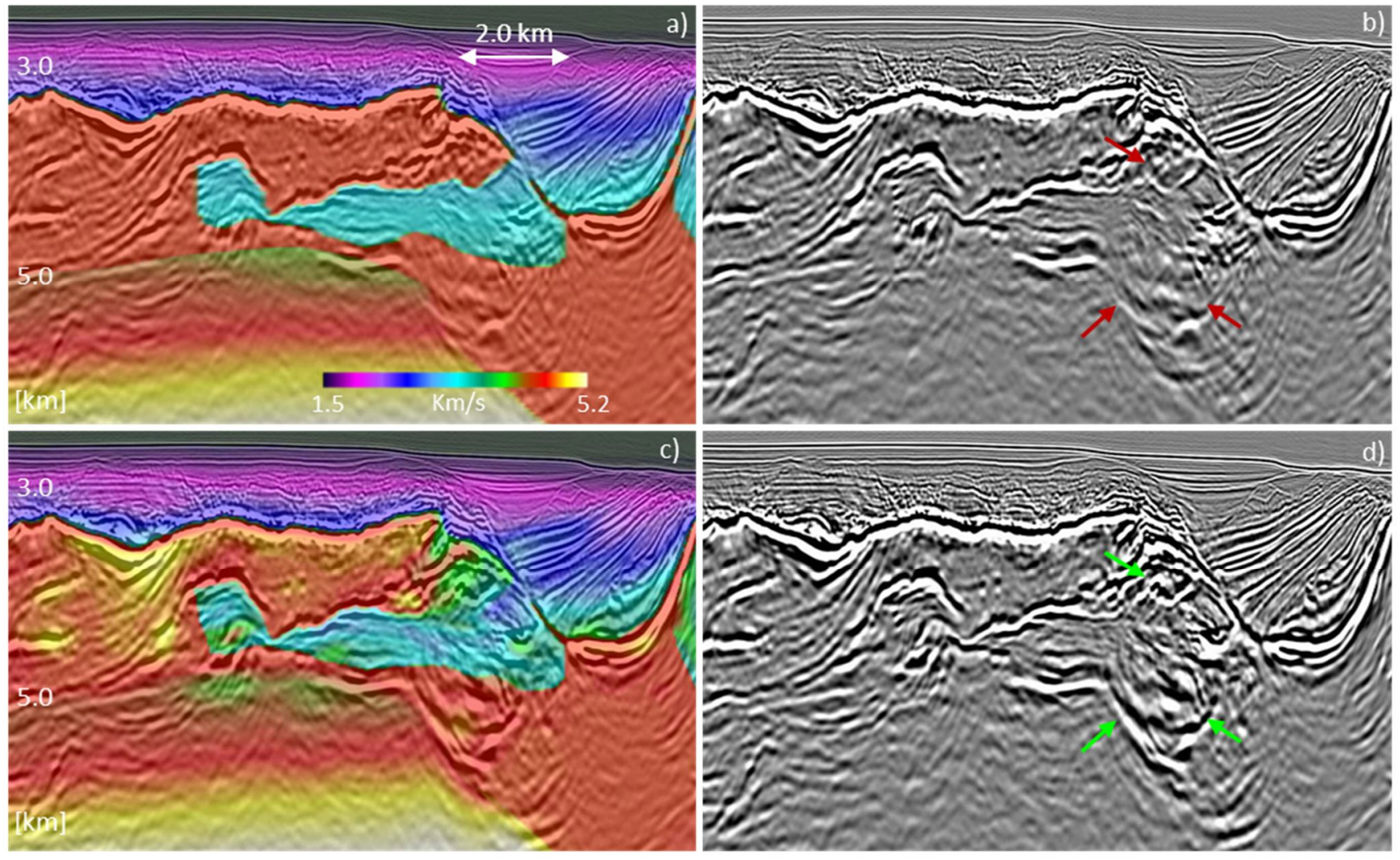

Figure 4 - Section comparing the legacy model buiding with the results of the iterative model building using TLFWI and stackpowrr WEMVA: (a) legacy velocity and (b) the corresponding RTM stack image; (c) updated velocity model and (d) RTM migration after 2nd iteration of TLFWI and stack-power WEMVA. The update suggests a change in the salt shape.

TLFWI and stack-power WEMVA, more improvements are noticeable in Figure $3 \mathrm{~h}$. As an example, the base of salt appears more focused below the mini-basin in the central part of Figure 3h.

\section{Discussion}

We showed that wave-equation based methods such as TLFWI and stack-power WEMVA better cope with complex geologies like those in some parts of the Santos basin. However, the availability of only NAZ data with limited offsets and low-frequency components still represents a big challenge for velocity model building.

After the second pass of our iterative approach, we observe some areas where the velocity update has significantly modified the initial velocity model and consequently the migrated stack image. As an example, let us consider the section shown in Figure $4 a$ and $4 b$, which lies in an area with a very big overhang below a salt wing. The legacy velocity model is shown in Figure 4a. We can note that the quality of the legacy image beneath the salt wing is very poor (Figure 4b), suggesting some unresolved salt complexities either at the level of the salt wing or in the welded area close to the base of salt. After the proposed iterative approach, we can note that the velocity model has a significant slowdown in one part of the salt wing (Figure 4c). The migrated image (Figure 4d) has a significant uplift in the same area. However, our approach was not able to fully resolve the complexity of the salt wing. This is probably due to the limitations of the available NAZ data.

Large velocity updates within the salt may suggest the need for some salt reinterpretation. Indeed, we could use the model inverted by the proposed approach as a guide for reshaping the salt. Then, after salt reinterpretation, we could go forward with a further pass of TLFWI and stackpower WEMVA. The advantage of a step involving manual salt interpretation is that it could possibly help go beyond the capacity of current technologies, especially with limitations of NAZ acquisition data.

\section{Conclusions}

Velocity model building in areas of highly complex geology, like the Santos basin's field dataset shown here, remains one of the toughest steps of seismic processing. The use of wave-equation based technologies is fundamental in such complex areas. Herein, we proposed a flow using TLFWI for the velocity update of the shallower layers and stack-power WEMVA for the deepest ones. These two methods allow to mitigate the impact of amplitude discrepancy in the respective velocity inversions. From our results, we showed that these methods have provided some reasonable image uplifts. Further improvements are 
observed with the iterative application of the TLFWI / stackpower WEMVA flow.

Despite the presented results being valuable, NAZ data remain a challenge for the velocity update and imaging of such complex areas. NAZ data limitations could lead to poorly constrained model inversions and limit the capacity of current technologies to find a better velocity update. Manual human intervention is, therefore, still necessary, for example with some salt-shape reinterpretation guided by the obtained velocity updates.

\section{Acknowledgments}

The authors would like to thank CGG for the permission to publish this work and CGG Multi-Client \& New Ventures for the Santos basin data set.

\section{References}

DONNO, D., S. BARRAGAN, F. JOUNO \& A. KHALIL, Improving pre-salt targets using wave-equation velocity analysis: submitted to $16^{\text {th }}$ International Congress of the Brazilian Geophysical Society, 2019.

GOMES, A. \& N. CHAZALNOEL, Extending the reach of FWI with reflection data: Potential and challenges: 87th Annual International Meeting, SEG, Expanded Abstracts, 1454-1459, 2017.

GOMES, A. \& Z. YANG, Improving reflection FWI reflectivity using LSRTM in the curvelet domain: 88th Annual International Meeting, SEG, Expanded Abstracts, 1248-1252, 2018.

IRABOR, K. \& M. WARNER, Reflection FWI: 86 $6^{\text {th }}$ Annual International Meeting, SEG, Expanded Abstracts, 11361140, 2016.

LUO, Y. \& G. T. SCHUSTER, Wave-equation traveltime inversion: Geophysics, 56(5), 645-653, 1991.

MA, Y. \& D. HALE, Wave-equation reflection traveltime inversion with dynamic warping and hybrid waveform inversion: 83rd Annual International Meeting, SEG, Expanded Abstracts, 871-876, 2013.

PRATT, R. G., Seismic waveform inversion in the frequency domain, Part 1: Theory and verification in a physical scale model: Geophysics, 64(3), 888-901, 1999.

SUN, D., K. JIAO, X. CHENG \& D. VIGH, Reflection based waveform inversion: 86th Annual International Meeting, SEG, Expanded Abstracts, 1151-1156, 2016.

SOUBARAS, R. \& B. GRATACOS, Velocity model building by semblance maximization of modulated-shot gathers: Geophysics, 72(5), U67-U73, 2007.

XU, S., D. WANG, F. CHEN, Y. ZHANG \& G. LAMBARÉ, Full waveform inversion for reflected seismic data: 74th
Annual International Conference and Exhibition, EAGE, Extended Abstracts, W024, 2012.

WARNER, M. \& L. GUASCH, Adaptive waveform inversion: Theory: Geophysics, 81(6), R429-R445, 2016.

ZHANG, Z., J. MEI, F. LIN, R. HUANG \& P. WANG, Correcting for salt misinterpretation with full-waveform inversion: 88th Annual International Meeting, SEG, Expanded Abstracts, 1143-1147, 2018. 